\title{
AVANCES EN LA CONSTRUCCIÓN DE OBRAS DE FÁBRICA
}

\author{
(BUILDING PROGRESS WITH MASONRY WORKS)
}

\author{
Walter Mann, Profesor Dr. Ingeniero \\ Lehrstuhl für Statik der Hochbaukonstruktionen Technische Hochschule Darmstadt Lichtwiesse \\ ALEMANIA
}

Fecha de recepción: 7-V-92

$631-7$

\begin{abstract}
RESUMEN
Los avances experimentados en la construcción de las fábricas en los últimos años en Alemania, después de analizados y ensayados en profundidad, permiten prever un crecimiento en la utilización de las fábricas, aprovechando las cualidades de sus materiales.

Se hace un amplio recorrido por los aspectos a tener en cuenta en las fábricas resistentes armadas y sin armar, considerados a partir de la normativa DIN, resaltando los más significativos.
\end{abstract}

\section{SUMMARY}

The building progress with masonry works in the last few years in Germany, after being analyzed and tested thoroughly, allow the prediction of an increasing use of masonries making the most of their material properties.

The article gives a wide review in the aspects of load bearing reinforced and non-reinforced masonries, considered through the Din regulation, with the emphasis on the most significant.
En los últimos tiempos, la construcción de fábrica vuelve a ganar importancia. Pasadas las épocas del hormigón visto o del hormigón lavado, se vuelve a recurrir a la primitiva técnica de la edificación, aprovechando sus ventajas como se describen a continuación.

Las pequeñas dimensiones de los elementos responden a nuestras escalas humanas y nos permite mayor flexibilidad en el proyecto y ejecución; la superficie nos trasmite una sensación de calor más intensa que la de los demás materiales, y un solo elemento, el muro de fábrica, satisface bien y económicamente la combinación de varias condiciones como el cerramiento de espacios, la transmisión de cargas, la protección térmica, acústica y contra el fuego.

Esta tendencia se ha visto favorecida por una serie de nuevos avances, impulsados por la Industria y por los Centros de Investigación, que han sido puestos en forma adecuada para la práctica en Comisiones y Grupos de Normalización. Durante los últimos años, también la Cátedra del autor ha contribuido, en diversas formas, a estos avances.

Posiblemente cabrá preguntarse qué es posible desarrollar aún en una técnica constructiva que está sirviendo a la Humanidad desde hace milenios. Por eso se va a desarrollar seguidamente algunos puntos.

\section{MATERIALES}

\section{La pieza de fábrica}

El desarrollo en el campo de los materiales de fábrica se ha caracterizado, sobre todo, por los siguientes aspectos: mayor protección contra el calor, elaboración más económica y mayor resistencia.

Para mejorar la protección contra el calor, se han perfeccionado los materiales porosos, como el hormigón ce- 
lular, cerámica aligerada, KS-Yali, etc., cuyas buenas propiedades aislantes proceden precisamente de su bajo peso específico -más ligeros que el agua-. Al mismo tiempo aparecieron en el mercado los ladrillos perforados, cuyos alvéolos estaban contrapeados para prolongar el recorrido del calor aumentando, al mismo tiempo, el número de perforaciones. Estos avances, positivos en cuanto al aislamiento térmico, tienen algunos inconvenientes si se consideran desde otros puntos de vista: al reducirse el peso empeora el aislamiento acústico, de modo que hay que prestar una especial atención a las consecuencias desde el punto de vista de la Física Aplicada a la Edificación; por otra parte, la distribución de las perforaciones reduce las resistencias a tracción, a compresión y tangencial de las piezas en dirección horizontal. Han llegado a medirse, en casos extremos, resistencias a compresión longitudinal de sólo el $1 \%$ de la resistencia en dirección vertical. Por lo tanto, queda aún algo que hacer en este aspecto.

Para poder colocar los bloques, con escaso empleo de mano de obra, se han desarrollado piezas de formato grande, pero esto aumenta también el peso de las mismas. Las entalladuras de agarre practicadas en los bloques facilitan su manejo. Para la colocación de los bloques más pesados, se encuentran en el mercado aparatos especiales que actúan como pequeñas grúas flexibles.

Se han conseguido también mayores resistencias a compresión de las piezas. Los ladrillos usuales hasta ahora correspondían a la Clase de resistencia 28; las normas recogen ahora ladrillos hasta la Clase 60 (DIN 1053).

\section{Morteros de albañilería}

Los morteros para albañilería también han experimentado un avance considerable. Aparte del aumento de resistencia a compresión (Morteros del Grupo IIIa), hay que mencionar especialmente los morteros ligeros (para mejorar el aislamiento térmico), los morteros de capa delgada y los morteros prefabricados. Todos los tipos de morteros pueden adquirirse hoy como prefabricados en instalaciones industriales, con lo que se consigue la simplificación de los trabajos a pie de obra y un incremento de la calidad. Por la importancia que tienen todos los tipos de morteros citados, y por las experiencias recogidas en la práctica, han sido incluidos recientemente en las normas.

\section{Organización de la obra de fábrica}

Hasta ahora se seguía la regla de que había que colocar mortero tanto en las llagas como en los tendeles. La aplicación del mortero en las llagas era muy laboriosa y, en muchos casos, se hacía mal. Los estudios teóricos y los ensayos prácticos realizados han demostrado que pueden dejarse las llagas en seco sin perjuicio desde el punto de vista estático, siempre que la anchura de la llaga sea muy pequeña, es decir, que no exceda de $3 \mathrm{~mm}$. Las normas recogen ahora estas fábricas de bloque construidas "en seco".

\section{DIMENSIONAMIENTO DE LAS OBRAS DE FÁBRICA}

\section{Normalización}

Las reglas sobre las obras de fábrica se reunieron, hace más de 50 años, en la Norma DIN 1053. Esta Norma se ha revisado varias veces. En la actualidad se divide en 4 partes, de las que se dispone en forma completa desde hace 2 años:

DIN 1053, la 1. Parte es la norma básica para obras de fábrica. Contiene las reglas para el cálculo y ejecución, especialmente un método de dimensionamiento simplificado, hecho a la medida para la práctica (1). Las demás se basan en la 1. Parte completando ésta. Así, por ejemplo, la 2 a Parte contiene un método de dimensionamiento más exacto, del cual se deriva el método simplificado, y que puede servir de ayuda en cuestiones más complicadas (2). La 3 a Parte da normas complementarias para la fábrica armada (3); y la 4 . $^{\text {a }}$ Parte trata de los elementos prefabricados de fábrica. Se dispone así de un conjunto de normas completo y aplicable a la práctica para este importante material de construcción.

\section{Solicitación a compresión de la fábrica}

En el marco de la revisión de la Norma DIN 1053 también se comprobó la resistencia a compresión de la fábrica. El Profesor Kitschig, de Hannover, recogió los resultados de más de 2.000 ensayos, con los que se verificaron y corrigieron las tensiones hasta ahora admisibles. Otros trabajos publicados se basan en ése. Así, por ejemplo, en (4) se desarrolla la resistencia del muro $\beta_{w}$ en forma completa, conforme a la figura 1 , en función de la resistencia del labrillo $\beta_{\text {ST }}$ y de la resistencia del 


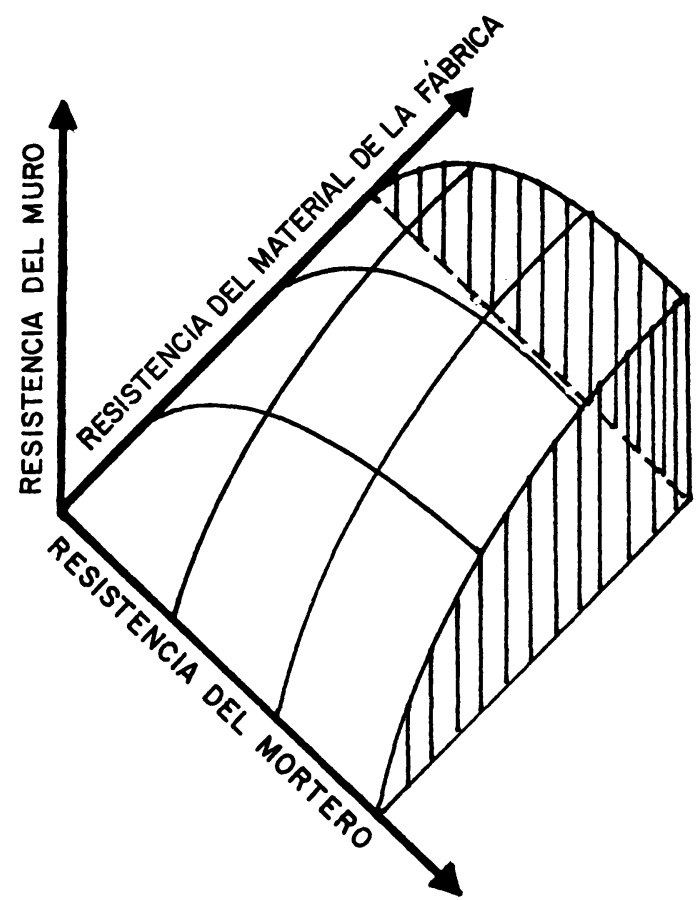

Fig. 1.-Resistencia de la fábrica $\beta_{w}$ en función de la resistencia del ladrillo $\beta_{S t}$ y de la resistencia del mortero $\beta_{M \ddot{o}:} \beta_{w}=a \cdot \beta_{S t}^{b} \cdot \beta_{M \ddot{c}}^{c}$

mortero $\beta_{\mathrm{M} \delta}$. En otros trabajos se han seguido las leyes mecánicas de la resistencia a compresión y así se hizo en la tesis desarrollada en la Cátedra citada (5). En la actualidad admitimos que, a causa de los diferentes comportamientos del ladrillo y del tendel de mortero en cuanto a alargamiento transversal, se originan tensiones de coacción que actúan sobre el ladrillo en forma de tracción transversal, lo fisuran y provocan la rotura.

Importantes estudios son los que se refieren al comportamiento a pandeo y el efecto de empotramiento entre muros y forjados (6). Los resultados obtenidos permitieron desarrollar nuevas hipótesis de dimensionamiento que, en muchos casos, permiten construir muros más esbeltos. De esta forma se puede prescindir con frecuencia de la necesidad de muros transversales de rigidización, con lo que el arquitecto puede actuar con mayor flexibilidad en los proyectos. Estas mismas hipótesis permiten captar con mayor exactitud el peligro de fisuración en los nudos muro-forjado y, en su caso, adoptar las medidas preventivas necesarias.

\section{Solicitación a tracción de la fábrica}

La resistencia a tracción de la fábrica es muy reducida, tanto en la dirección vertical como en la horizontal. Fre-
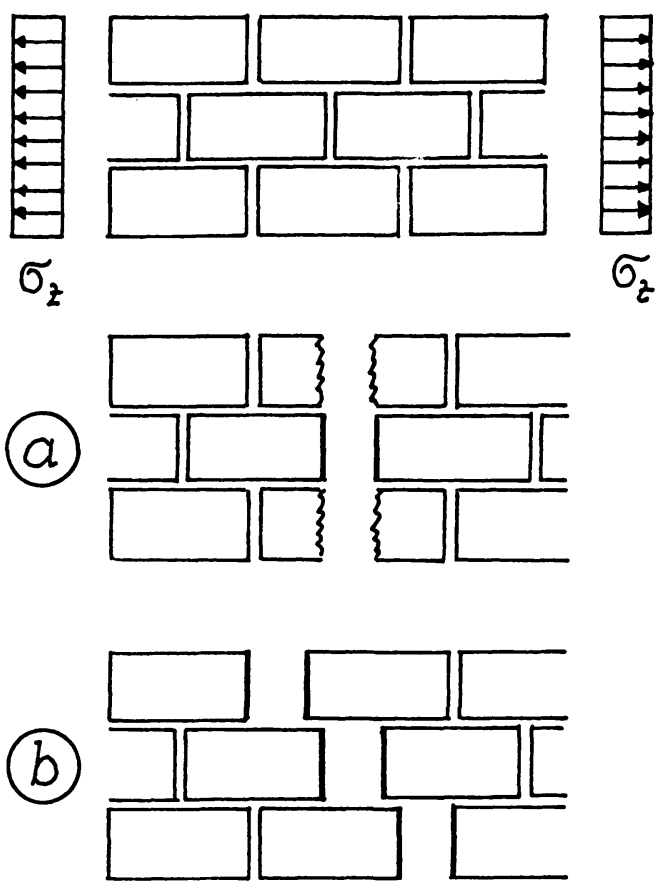

Fig. 2.-Resistencia a tracción de la fábrica si: a) hay rotura de la pieza; b) hay rotura de las juntas.

cuentemente se debe, bien a las coacciones de retracción, o a las acciones térmicas, por lo cual hay que ser muy cautos en la utilización de esta resistencia para la absorción de cargas. También se han realizado numerosos ensayos en este sentido, que permitieron basar en ellos otros estudios analíticos como los recogidos en (7). El trasfondo mecánico es el conocimiento de que, según la figura 2, pueden producirse dos tipos de rotura:

a) Si la resistencia a tracción de la pieza es escasa y la trabazón de la fábrica buena, las fisuras afectarán a las piezas partiendo de las llagas.

b) Sin embargo, si la resistencia a tracción de las piezas es grande y la trabazón o el rozamiento del mortero de las llagas son malos, fallará la trabazón aunque los ladrillos no lleguen a fisurarse.

Las hipótesis de cálculo recogen este mecanismo, por lo que podemos predecir con mayor exactitud el comportamiento del muro.

\section{Resistencia a corte de la fábrica}

Los muros no sirven solamente para absorber las acciones verticales, sino también para la rigidización horizontal 


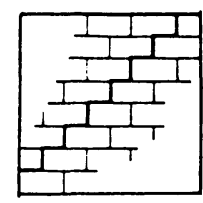

(a) (b)

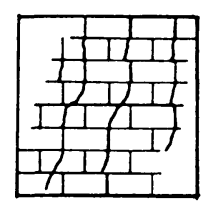

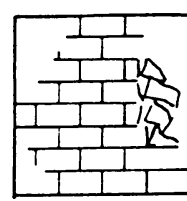

(c)

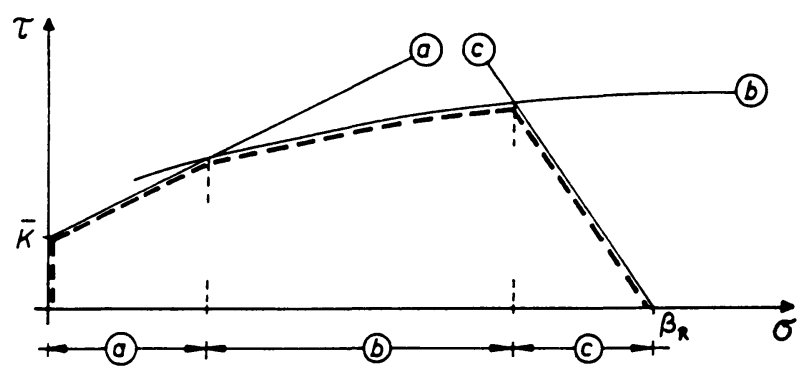

Fig. 3.-Resistencia a corte de la fábrica si: a) hay rotura de las juntas; b) se fisuran las piezas; c) hay rotura a compresión.

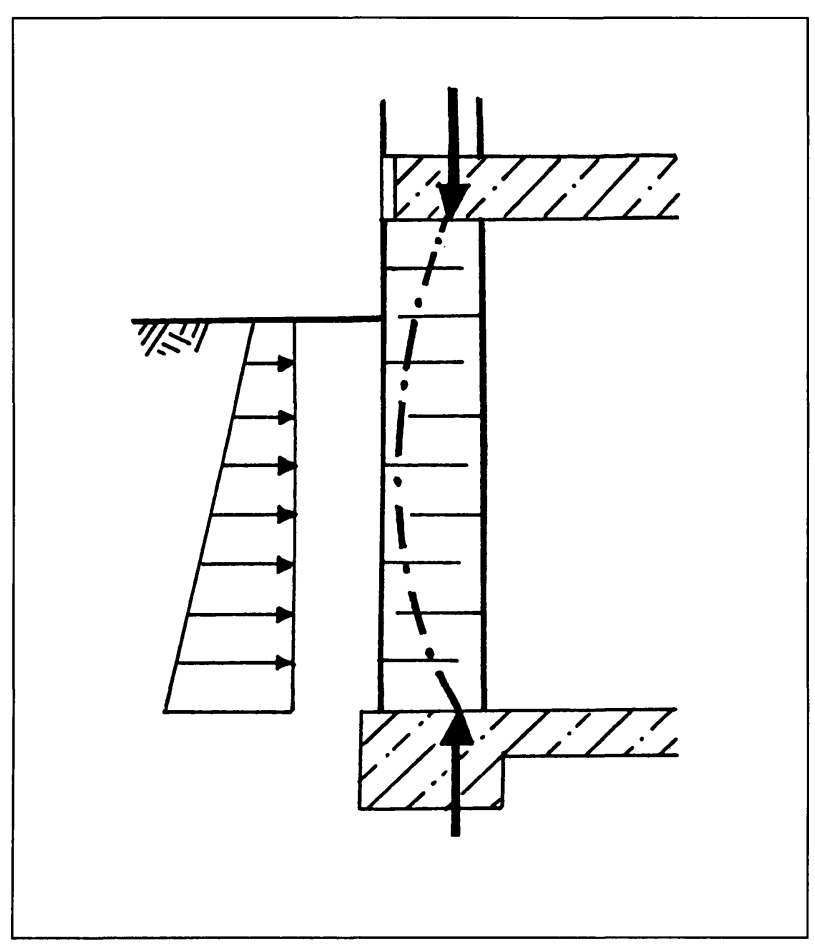

Fig. 4.-Curva de compresiones en un muro de sótano sometido a empuje del terreno.

y para absorber las acciones eólicas. De ahí proceden las tensiones de corte. El comportamiento de la fábrica en este último caso se recogió en una teoría sobre la resistencia a cortante, la cual se ha podido verificar y confirmar mediante numerosos ensayos (8). En este caso hay que distinguir diversos tipos de rotura representados en la figura 3.

Si la adherencia en los tendeles es escasa, pero buena la resistencia a corte de las piezas, fallará la trabazón, es decir, aparecerán fisuras escalonadas en las llagas y tendeles manteniéndose intactas las piezas. Si la resistencia a corte de las piezas es escasa y la adherencia en las juntas es buena, las fisuras afectarán a las piezas. Y si las acciones verticales son muy intensas, puede también producirse la rotura del muro de fábrica por compresión.

Este tipo de solicitación está adquiriendo cada vez mayor importancia. Por una parte, la altura de los edificios que se construyen suele ser mayor, con el consiguiente aumento de las solicitaciones eólicas (son ya raros los edificios de 10 a 15 plantas con cerramientos de fábrica de ladrillo). Por otra parte, es frecuente tener que ejecutar proyectos en que se dispone de pocos muros de rigidización, por lo que los distintos muros pueden resultar sobrecargados, incluso en edificios bajos.

\section{PUNTOS DE VISTA CONSTRUCTIVOS}

\section{Muros de sótano}

Hasta ahora, los muros de sótano tenían que construirse con piezas de la Clase de Resistencia 4 como mínimo. Esta limitación ha desaparecido recientemente, ya que era evidente que se había adoptado más bien "a estima" y no podía darse ninguna razón convincente para ello. Por el contrario, había un gran interés en utilizar en el sótano materiales con más capacidad de aislamiento térmico.

En muchos casos, el empuje del terreno sobre los muros de sótano constituye un problema. Como la resistencia a tracción de la fábrica es deficiente, el momento flector resultante sólo puede absorberse mediante una sobrecompresión. Puede imaginarse esto como la acción de una curva de compresión trazada verticalmente dentro del espesor del muro, tal como se representa en la figura 4. La capacidad resistente del muro también depende, en este caso, de que la carga vertical aplicada sea suficiente. En (9) se ha estudiado más detenidamente este caso y se ha desarrollado un método simplificado de dimensionamiento que permite captar con mayor exactitud y, en parte, también más favorablemente las condiciones de trabajo del muro. Se continúan ahora los estudios sobre la transmisión biaxil de las cargas (10). Es apremiante disponer de nuevos descubrimientos en este campo. 


\section{Muros de dos hojas}

El incremento de las exigencias impuestas al aislamiento térmico nos lleva, cada vez más, a los muros exteriores de dos hojas. Los problemas inherentes a este sistema son múltiples: anclaje entre ambas hojas, protección contra la corrosión de los anclajes, tipo de aislamiento térmico, por ejemplo, aislamiento central con el espacio que necesita, la distancia necesaria entre juntas de dilatación y su absorción en dirección vertical, absorción en la zona de antepechos, etc. Aunque ya se ha hecho mucho en este campo, tanto en teoría como en ensayos, y aunque la nueva redacción de las normas incluye simplificaciones y precisiones, los muros de dos hojas siguen constituyendo, hoy como ayer, un amplio campo de estudio.

\section{FÁBRICA ARMADA}

Desde hace 2 años se dispone (con la Norma DIN 1053, 3 ? Parte) de una amplia normalización para la fábrica armada, por lo que hay que suponer que se irá aplicando esta forma de construcción con mayor frecuencia, ya que hasta ahora apenas tiene aplicación entre nosotros. La Norma contiene las reglas esenciales para el dimensionamiento y ejecución de la fábrica armada para la absorción de solicitaciones. No se ha pensado exclusivamente en una armadura que sólo sirva para evitar la fisuración (para eso se dispone ya de las recomendaciones de las Empresas). En (11) y (3), así como en las figuras 5 a 8 , se pueden encontrar aclaraciones y ejemplos al respecto.

El principal problema lo constituye actualmente la protección de las armaduras contra la corrosión. Los ensayos realizados han demostrado que las juntas de mortero se carbonatan totalmente en poco tiempo, por lo que desaparece la atmósfera básica en el mortero y, por lo tanto, la protección contra la oxidación. Por esta razón, la armadura resistente de acero desnudo sólo debe colocarse en muros sometidos a un ambiente predominantemente seco en el interior de los locales. En los muros exteriores se precisa de medidas complementarias como: acero inoxidable, revestimiento de epoxi galvanizado fuerte, etc. Aparte del problema del precio, se plantean otras cuestiones: propensión a lesiones en obra, tensiones de adherencia con el mortero, tolerancias de puesta en obra, formación de uniones de esquina y empalmes de las armaduras, etc. La firma BEKAERT ha desarrolla-

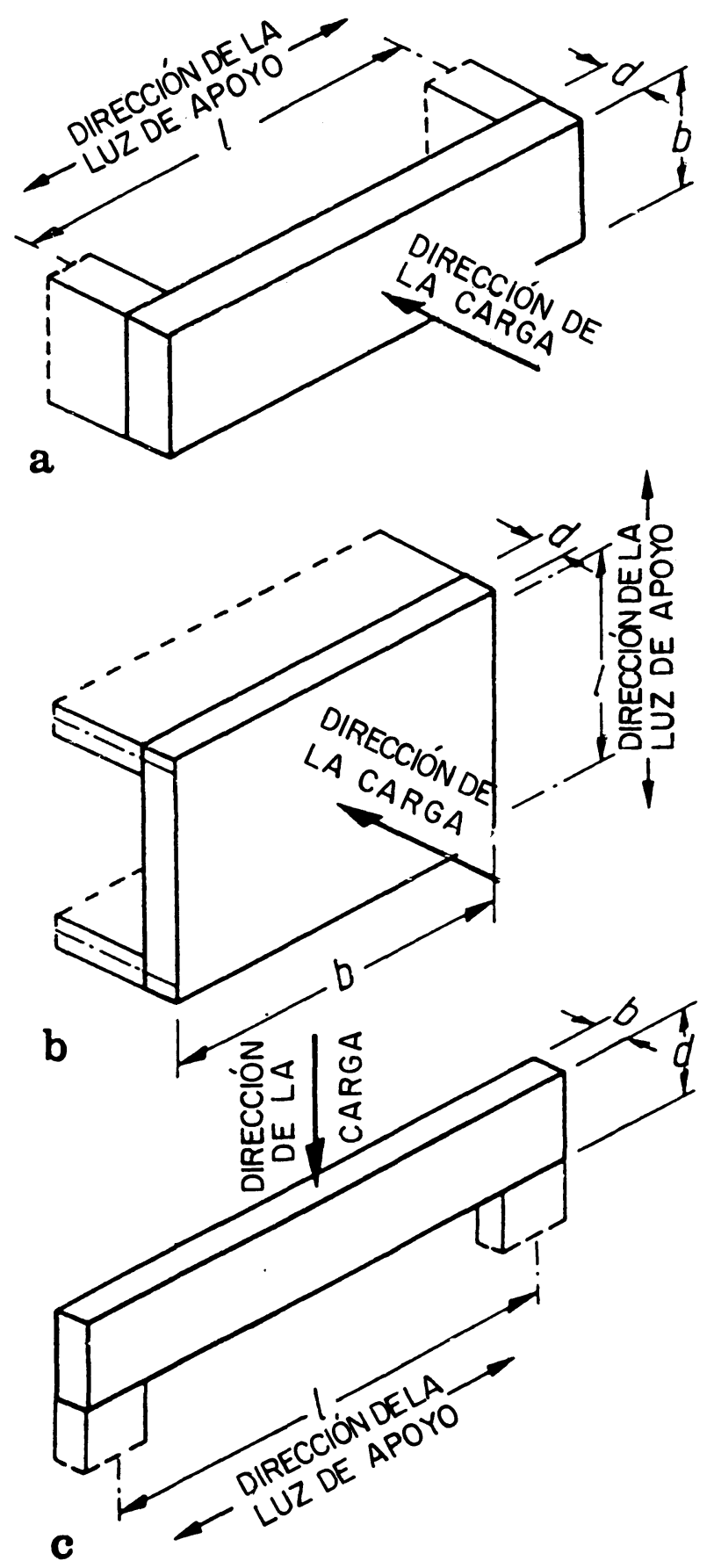

Fig. 5.-Aplicaciones de la fábrica armada: a) Efecto resistente de placa con luz horizontal entre apoyos verticales; b) Efecto resistente de placa con luz vertical entre apoyos horizontales; c) Efecto resistente de viga de canto.

do unos elementos de armado prefabricado (Murfor) de redondo de $5 \mathrm{~mm} \varnothing$ atados entre sí, que pueden colocarse en los tendeles (Figura 6). Pueden adquirirse en versión ligeramente galvanizada y también con recubrimiento adicional de epoxi. Se ha solicitado la homologación de este último, por lo que se podría utilizar tanto para muros interiores como para exteriores. Las figuras 5 a 8 representan diversos ejemplos de aplicación. Se 


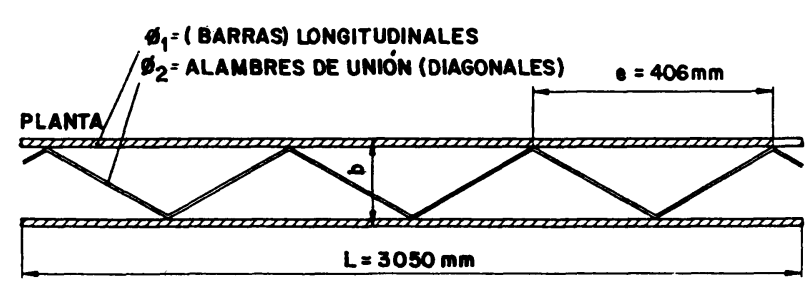

$\sigma_{1}=$ (BARRAS) LOMVITUDINALES BST 500/550 RK, CORRUGADAS DE $5 \mathrm{~mm}$ DE DIAMETRO

$\sigma_{2}=A L A M B R E S$ DE UNIÓN, SUPERFICIE LISA, DE $3,75 \mathrm{~mm}$ DE DIAMETRO $L=$ LONGITUD DE CADA ELEMENTO $3,050 \mathrm{~m}$ (LONO.ESTANDAR) $06,100 \mathrm{~m}$ $b=$ ANCHURA DEL ELEMENTO

Fig. 6.-Armadura prefabricada Murfor para colocar alambres en los tendeles.

a

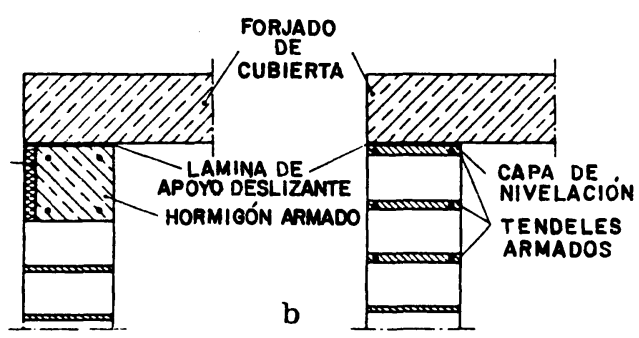

Fig. 7.-Zunchos perimetrales: a) de hormigón armado; b) de fábrica armada.

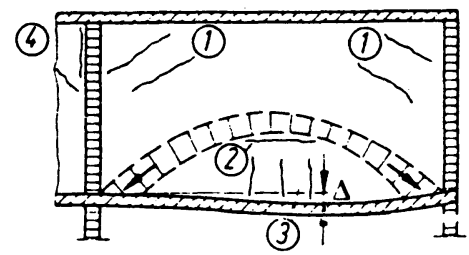

(1) Fisuras diagonales (2) FISURAS HORIZONTALES

(3) FisURAS VERTICALES EN ZONA CENTRAL INF. (4) FISURAS VERTICALES
JUNTO A SOPORTES

Fig. 8.-Fisuración en paredes divisorias como consecuencia de la flexión del forjado.

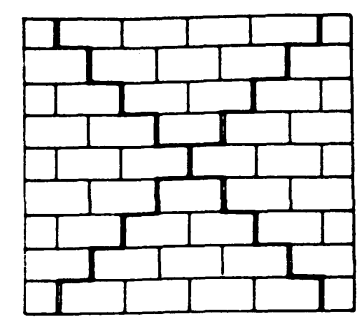

(a)

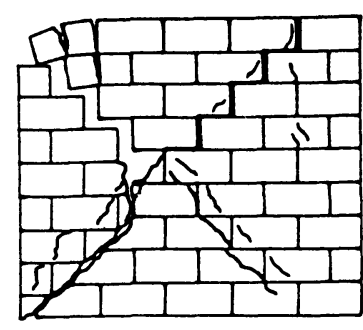

(b)
Fig. 9.-Forma de las fisuras en muros como consecuencia de las acciones sísmicas con: a) rotura de las juntas; b) fisuración de las piezas de la fábrica. supone que pronto se aplicarán, en especial, los zunchos perimetrales de fábrica armada, que evitarán los inconvenientes de los zunchos normales de hormigón armado (cambio de material, condiciones físicas, tiempo necesario, precio) (Figura 7). También es posible que se abra paso el armado constructivo de muros no resistentes para evitar la fisuración, tan pronto como el principio de la fábrica armada sea conocido en un ámbito más extenso.

\section{FÁBRICA EN ZONAS SÍSMICAS}

Se discute mucho la viabilidad de la fábrica en zonas sísmicas. En nuestro país no hemos tenido malas experiencias en este aspecto, siempre que se han seguido las reglas básicas de la construcción en zonas sísmicas, especialmente en cuanto a rigidización suficiente y lógica y la elección adecuada de los materiales. Por esta razón estamos interesados en mantener esta forma de edificación.

Para obtener mejores conocimientos sobre este tema, en colaboración con el Prof. König de la ETS de Darmstadt, se han realizado ensayos en una mesa vibratoria. Se utilizaron muros de ensayo de distintos tipos que se sometieron a solicitaciones sísmicas simuladas. Los ensayos (12) dieron los resultados expuestos a continuación.

Las reglas conocidas para las solicitaciones estáticas por la teoría del cizallamiento pueden utilizarse también para las solicitaciones dinámicas. Es importante que las calidades de la pieza y del mortero se coordinen entre sí de modo que se produzcan fisuras escalonadas en las juntas, pero sin fisuración de las piezas (Figura 9). Si es así, la energía queda absorbida por el rozamiento en las juntas, mientras que las acciones verticales siguen transmitiéndose por medio de los tendeles. En cambio, si aparecen fisuras en los ladrillos, se forma una especie de curva de deslizamiento con rotura total súbita. Se vio que son poco apropiadas las piezas perforadas con alvéolos contrapeados en la dirección de las fuerzas, ya que actúan con poca homogeneidad en esa dirección y pueden originar también la rotura súbita. Es de esperar que se difundan más los conocimientos de la forma correcta de construir en zonas sísmicas, y que en ellas mantenga la fábrica el lugar que le corresponde. 


\section{AVANCES EN EL MARCO DE LA NORMALIZACIÓN EUROPEA}

La armonización de las normas de la edificación a que se tiende en Europa, comprende asimismo la construcción con fábrica. Desde hace años están trabajando grupos internacionales, cuyo trabajo resulta evidentemente difícil, laborioso y fatigoso. Los resultados que, hasta este momento, pueden resultar conocidos, se refieren a los puntos siguientes: espesor mínimo de muros; aumento del aprovechamiento de las resistencias aplicando una distribución no lineal de las tensiones; comprobación de la seguridad no por medio de las tensiones admisibles, sino por medio de coeficientes de seguridad parciales; ampliación de la admisibilidad de las tensiones a tracción, y otros muchos puntos de detalle. Los proyectos de normalización actuales son documentos de compromiso y bastante voluminosos, así como insólitos y poco manejables para los profesionales en muchos aspectos. No es previsible aún la finalización de estos trabajos; en todo caso no podrá disponer de ellos antes del fin de este decenio. En ese momento quedarán anuladas las Normas DIN que ya no son conocidas y serán sustituidas por el nuevo Euro-Código.

\section{LA OBRA DE FÁBRICA COMO MATERIAL DE CONSTRUCCIÓN SUSCEPTIBLE DE DESARROLLO}

Se ha presentado una serie de avances en la construcción de fábrica, que no podía ser completa debido a la brevedad de esta exposición. Volviendo atrás, a la cuestión planteada al principio, de si en este material tan antiguo aún queda algo por desarrollar, y hay que concluir diciendo que las fábricas, a pesar de su gran antigüedad, siguen siendo, hoy como ayer, un material importante, valioso y de gran interés. Sigue siendo joven para los profesionales y mantiene su interés, precisamente por su capacidad de desarrollo.

\section{BIBLIOGRAFÍA}

(1) MANN, W., Grundlagen der vereinfachten Bemessung von Mauerwerk nach DIN 1053, Tel 1 (Fundamentos del dimensionamiento de las obras de fábrica según DIN 1053, 1. a Parte). Mauerwerk-Kalender 1988.
(2) MANN, W., Grundlagen der ingenieurärigen Bemessung von Mauerwer nach DIN 1053, Teil 2 (Fundamento del Cálculo de Ingeniería de la fábrica según DIN 1053 2. Parte). Mauerwerk-Kalender 1992 y anteriores.

(3) MANN, W. Y ZAHN, J., Bewehrung von Mauerwer zur Rissesicherung un Lastabtragund (Armado de la fábrica contra la fusuración y para la absorción de cargas). Mauerwek-Kalender 1990.

(4) MANN, W., Durckfestgkeit von Mauerwerk-eine statistische Auswertung... mit Hilfe von Potenzfunktionen (Resistencia a compresión de la fábrica -análisis estadísticocon ayuda de funciones potenciales). Mauerwek-Kalender 1983.

(5) SCHULENBERG, W., Theoretische Untersuchung zum Tragverhalten von zentrisch gedrückten Mauerwerk... Dissertation (Estudio teórico sobre el comportamiento resistente de la fábrica sometida a acciones de compresión centradas... Tesis). Darmstadt 1982.

(6) MANN, W. y LEICHER, E., Untersuchungen zum Nachweis der Knicksicherheit unter Berücksichtigung der Deckeneinspannung (Estudios para comprobar la seguridad al pandeo de los muros de fábrica, teniendo en cuenta el empotramiento de los forjados). Mauerwek-Kalender 1986.

(7) MANN, W., Zug-und Biegezugfestigkeit von Mauerwek -theoretische Grundlagen und Vergleich mit Versuchsergebnissen (Resistencia a tración y flexotracción de la fábrica-. Fundamentos teóricos y comparación con los resultados de ensayos). Mauerwek-Kalender 1992.

(8) MANN, W., MUILLER, H., Schubtragfähigkeit von gemauerten Wänden... (Capacidad resistente al cizallamiento de los muros de fábrica....). Mauerwek-Kalender 1985.

(9) MANN, W., y BERNHARDT, G., Rechnerische nachweis von gemauerten Wänden, insbesondere von Kellerwänden, auf Erddruck (Cálculo analítico de muros de fábrica, especialmente muros de sótano, sometidos al empuje de las tierras). Mauerwek-Kalender 1984.

(10) MANN, W., y TONN, V., Das Tragverhalten von zweiachsig gespannten gemauerten Wänden unter gleichzeitg wirkender horizontaler und vertikaler Belastung (Comportamiento resistente de muros de fábrica empotrados biaxilmente bajo la acción simultánea de cargas horizontales y verticales). Mauerwek-Kalender 1989.

(11) MANN, W., y ZAHN, J., Bewehrtes Mauerwerk zur Lastabtragung und zur konstruktiven Rissesicherung. Ein Leitfaden für die Prraxis (Fábrica armada para absorción de cargas y seguridad constructiva contra la fisuración. Una guía para la práctica). Bekaert, S. A. 1991.

(12) MANN, W., KÖNIG, B., y ÖTSCH, A., Versuche zum Verhalten von Mauerwerk unter seismischer Beansprunchung (Ensayos sobre el comportamiento de la fábrica sometida a acciones sísmicas), Mauerwek-Kalender 1989. 